\title{
L'AVIS AU LECTEUR DU RECUEIL DE QUELQUES PIÈCES CURIEUSES (1684)
}

\author{
Jean-Robert Armogathe* \\ armogathe@wanadoo.fr
}

RESUMO O Recueil de pièces publicado por Bayle em 1684 tem sido objeto da atenção dos historiadores do cartesianismo : nós propomos aqui o estudo do paratexto bayliano ("Nota ao leitor, escolha e apresentação dos textos »), revelador de uma estratégia de controvérsia histórica e literária produtora do essencial de sua potência.

Palavras-chave Bayle, Descartes, cartesianismo, Santo Oficio, Le Valois (Louis), Lanion (François de)

RÉSUMÉ Le Recueil de pièces publié par Bayle en 1684 a retenu l'attention des historiens du cartésianisme: nous nous attachons ici à l'étude du paratexte baylien (avis au lecteur, choix et présentation des textes), qui est révélateur d'une stratégie de controverse tirant de la critique historique et littéraire l'essentiel de sa puissance.

Mots clés Bayle, Descartes, cartésianisme, Saint-Office, Le Valois (Louis), Lanion (François de)

Le Recueil de quelques pièces curieuses concernant la philosophie de M. Descartes, paru chez Henri Desbordes à Amsterdam en 1684, a davantage retenu l'attention des spécialistes de Descartes que de Bayle (il a été republié

* École Pratique des Hautes Études, Paris. Recebido em 04/09/2009 e aceito em 21/09/2009.

KRITERION, Belo Horizonte, nº 120, Dez./2009, p. 449-460. 
au XIX ${ }^{\mathrm{e}}$ siècle dans un recueil d'Cuvres philosophiques de Descartes), ${ }^{1}$ mais les historiens de la philosophie cartésienne se sont plus attachés à étudier les pièces publiées que leur éditeur. Reprenant ce dossier après plus de quarante ans, je voudrais le présenter comme une glose de l'excellente étude de Hubert Bost sur "diffusion et discussion du cartésianisme", qui a pour corpus principal les Nouvelles de la République des Lettres. ${ }^{2}$

Il s'agit bien, comme l'indique le titre, d'un recueil de pièces, inédites ou publiées de façon clandestine, introduit par un avis au lecteur, que nous allons maintenant commenter.

Bayle souligne dès l'entrée la dimension clandestine: la plupart des pièces ont déjà été imprimées, mais elles sont restées cachées par peur des juifs - une expression fréquente dans le Nouveau Testament, surtout dans le quatrième Évangile -, c'est-à-dire, en l'espèce, par peur de la censure royale. Bayle ironise à partir de vers de Boileau repris pour la circonstance et légèrement modifiés: là où Boileau avait écrit:

Le Jonas ${ }^{3}$ inconnu sèche dans la poussière,

Le David ${ }^{4}$ imprimé n'a pas vu la lumière/

(Satire IX, 91),

Bayle contamine les deux vers, et il écrit “Le Jonas imprimé n'a pas vu la lumière" (peut-être influencé par la légende de Jonas dans le ventre du poisson).

Bayle présente ainsi son texte comme une apologie pour la liberté de la librairie. Il poursuit par une dénonciation du nouveau régime de la librairie: en fait, les historiens du livre s'accordent pour reconnaître qu'en matière de contrôle de la librairie, la période 1678-1701 fut certainement la plus rigoureuse que connut 1'Ancien Régime. ${ }^{5}$ Le lieutenant général de police, La Reynie, entre 1667 et 1697, était particulièrement attentif aux délits de librairie. Le commissaire de police du quartier de l'Université et du Palais, où se trouvaient la plupart des libraires parisiens, Nicolas de La Mare (16391723), a publié en un intéressant Traité de la police qui montre bien la rigueur

1 Descartes, René. CEuvres philosophiques, publiées par Adolphe Garnier. Paris, 1835. t. IV, p. 335-457.

2 Bost, Hubert. Pierre Bayle historien, critique et moraliste. Belgium : Turnhout, 2006. p. 69-85.

3 Jonas ou Ninive pénitente (1663) de Jacques de Coras; il est aussi question dans le Lutrin du « seul Jonas relié qui ait jamais vu le jour".

4 Jacques de Coras a aussi écrit un David, ou la vertu couronnée, mais c'est un autre poème religieux qui est ici visé, le David de David de Lasfargues, un Toulousain (d'après une remarque de Saint-Marc, éd. des CEuvres de Boileau, Amsterdam, 1772, t. I, p. 180).

5 Sauvy, Anne. Livres saisis à Paris entre 1678 et 1701. La Haye : M. Nijhoff, 1972. p. 5. 
de la surveillance des livres et la sévérité de la répression des clandestins. ${ }^{6}$ Mais, si les impressions clandestines étaient devenues rares, le public n'en recherchait pas moins les "mauvais livres": ils étaient imprimés en province (Lyon et Rouen), où la surveillance des imprimeurs était moindre, ou à l'étranger, où la police française ne pouvait rien faire (même si, par exemple en 1669, la cour de France envoya un commissaire en Hollande pour obtenir des États qu'ils interdisent les "méchants livres": 7 la guerre franco-hollandaise rendit ces mesures inopérantes). Anne Sauvy, en 1972, a édité et annoté les listes de saisies entre 1678 et 1701: on y trouve d'ailleurs sous le numéro 213 1'ouvrage que nous étudions.

Bayle ne manque pas de relever, parmi les personnes qui se plaignaient de la censure des livres, un docteur de Sorbonne, c'est-à-dire un des censeurs potentiels, Jacques Le Fèvre. ${ }^{8}$ Celui-ci avait repris Arnauld (sur la question de l'inamissibilité de la justice chez les Réformés), mais son manuscrit avait eu du mal à franchir le cap de l'approbation... Il s'en était plaint dans une lettre imprimée à M. Arnauld du 25 juillet 1683 (in- $4^{\circ}$ s.l.n.d. de 8 pages). Bayle s'en prévaut...

C'est au privilège du roi que Bayle s'en prend, c'est-à-dire qu'il ne s'agit pas seulement d'ouvrages soumis à approbation, comme les ouvrages traitant de la religion, mais de tous les livres qui avaient besoin du privilège valant autorisation de parution. Or, dit Bayle, les ouvrages de galanterie "qui ne peuvent que corrompre les bonnes mœurs des jeunes gens" échappent à cette sévérité, qui frappe "les bons livres, qui se donnent la liberté d'examiner les opinions généralement reçues, qui sont bien souvent les plus fausses". Le polémiste exagère: de nombreux livres libertins ont été saisis, mais la liste publiée par Anne Sauvy montre bien la proportion d'ouvrages saisis pour des raisons religieuses ou politiques, qui examinaient, précisément, les idées reçues.

Bayle passe ensuite à l'analyse des pièces réunies dans son recueil:

a) une espèce de concordat entre jésuites et oratoriens, où ceux-ci s'engageaient à ne plus enseigner la philosophie de Descartes. Bayle commente de manière sarcastique l'opposition des jésuites, "soit qu'ils craignissent que les collèges de philosophie où les pères de l'Oratoire régenteraient n'attirassent

6 T. I, 1705.

7 Sauvy, Anne. Ibidem, appendice IV. p. 426-430.

8 Sur lui (et ses démêlés avec Arnauld), voir l'abbé P. Féret. La Faculté de théologie de Paris et ses docteurs les plus célèbres. Époque moderne, t. VII: xvII siècle: Revue littéraire, Paris, 1910, p. 21-28. 
toute la jeunesse, qui trouve cent fois plus de goût à la nouvelle philosophie qu'à la vieille, soit qu'ils craignissent que les principes de Descartes ne fissent brèche à la religion". Et pour qu'on ne se méprenne pas sur l'intention satirique, il poursuit: “il y avait apparemment de l'un et de l'autre dans leur crainte, mais beaucoup plus du premier que du dernier".

Il s'agit de la seizième assemblée générale (et non: sixième) de l'Oratoire de France. Les "nouvelles opinions", fondées sur les ouvrages de Descartes, mais souvent éclectiques, inquiétaient le système universitaire dans son ensemble. Les débats qui eurent lieu à Louvain dans l'année 1662 aboutirent à un examen romain, puis à la mise à l'Index de plusieurs écrits latins de Descartes en 1663. Le 4 août 1671, l'archevêque de Paris, Harlay, signifie aux députés de l'Université de Paris un ordre verbal du roi: le contenu théorique est confus, puisqu'il y a fait allusion à la condamnation des propositions de Clave en 1624. ${ }^{9}$ Il souligne le risque de "quelque confusion dans l'explication de nos mystères", c'est-à-dire l'Eucharistie.

Le cartésianisme réapparaît dans les dossiers du Saint-Office et de l'Index romain quelques années après la condamnation de $1663,{ }^{10}$ lorsque, en 16711673, le père jésuite Honoré Fabri (1607-1688) avertit le secrétaire de la Congrégation :" "des théologiens français, explique-t-il, suivent la doctrine de Descartes et enseignent, selon le système philosophique de cet auteur, une nouvelle explication de la transsubstantiation, sur la permanence des accidents du pain sans sujet, qui renouvelle les erreurs de Bérenger et de Wiclef $»{ }^{12}$ Le secrétaire du Saint-Office, qui cite "la dottrina cartesiana", ne manque pas de souligner les différences, mais attribue au philosophe français une explication

9 Kahn, Didier. "La condamnation des thèses d'Antoine de Villon et Étienne de Clave contre Aristote, Paracelse et les 'cabalistes' (1624)", Revue d'histoire des sciences 55 :2 (2002) : 143-198; la Nouvelle lumière philosophique d'Ét. de Clave a été publiée par Bernard Joly (Paris: Fayard (coll. Corpus), 2000).

10 Les documents sont édités et annotés dans Armogathe, J.-R. et Carraud, Vincent. "La première condamnation des CEuvres de Descartes, d'après des documents inédits aux Archives du Saint-Office", Nouvelles de la République des lettres (Naples), 2001-II : 103-137.

11 Nous n'avons pas encore retrouvé ce document, qui semble être distinct de la censure que le jésuite Fabri, alors théologien de la Sacrée pénitencerie, avait rédigée en 1660 (BM Chartres, ms 366, p. 26-28; texte dans Sortais, G. Le Cartésianisme chez les jésuites français au xVII et au XVIII siècle. Paris : G. Beauchesne,

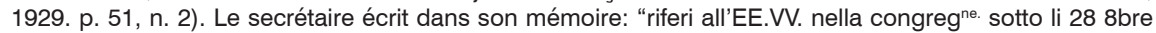
1671 come dal Pe Fabri Gesuita ero stato avvertito..."

12 Le secrétaire recopie la dénonciation de Fabri: “... (che) i Tologi (!) di Francia seguaci della dottrina di Renato di Cartes, havevano, secondo il sistema filosofico insegnato da questo autore, introdotto nuova maniera di spiegare come transustantiato per la forza delle parole della consacrazione il pane nel corpo di Christo Signore nostro, potessero senza soggetto rimanere gl' accidenti del pane istesso e che seguendo da simil dottrina a molti inconvenienti gravissimi et errori gia condannati da Nicolo papa nel Concilio romano nella causa di Berengario e dal Concilio di Costanza contro Wiclef, parea necessario che si applicasse dalle Sede apostolica qualche remedio prima che questa novità facesse più profondi radici”. 
atomiste. Cela peut sembler étrange, mais Cordemoy ou Heereboord se réclamèrent bien de Descartes pour construire un système atomiste.

Après l'entrevue de l'archevêque avec l'Université, dans une dépêche du 14 août $1671,{ }^{13}$ l'abbé de Vibò (qui assurait l'intérim de la nonciature) rapporte au Saint-Office les termes de l'entretien qu'il venait d'avoir avec François de Harlay (1625-1695) au début du mois d'août 1671: l'archevêque explique que s'il a agi contre les doctrines de Descartes, c'est "perche vedeva che si cominciava a far un partito di filosofi, che si chiamavano i Cartesiani, ${ }^{14} \mathrm{i}$ quali davano fuori tesi, facendo varie questioni sopra la materia, forma, accidenti e sostanza in una maniera che si conosceva benissimo, che l'intenzione loro era di combattere la transubstanzianzione dell'eucharistia perchè nelle Provincie d'Olanda oggidi non s'insegna altra filosofia, che quella di Descartes per gettar le prime impressioni nella gioventù in ordine alla sudetta opinione di negare la transubstanziazione".

L'archevêque dit aussi avoir informé le roi, avant son départ pour Fontainebleau. Le roi s'était alors déclaré opposé à cette nouvelle philosophie; un arrêt ayant déjà été pris, il souhaitait que des mesures fussent mises en œuvre, non seulement contre les professeurs, mais aussi contre les disciples. Le procureur du roi avait été avisé de procéder contre eux sans délai, en vertu dudit arrêt. Enfin, l'archevêque explique qu'il avait convoqué les professeurs, les avait avertis et avait obtenu d'eux la promesse de renoncer à enseigner de telles doctrines. ${ }^{15}$

Une grande agitation régnait à Louvain, où la Faculté de théologie, le 16 juin 1673, dénonçait encore auprès du maître du Sacré-Palais, le dominicain

13 Qui ne fut transmise de la secrétairerie d'Etat au Saint-Office que le 14 novembre; le Saint-Office répondit le 24 novembre à Vibò (notes manuscrites sur la dépêche). Cette dépêche est citée par le P. Raimondo Nidi, o.p., censeur d'un écrit antiaristotélicien en 1673 (voir plus bas, à propos de la Physica Antiperipatetica de Francesco Pasolino).

14 L'Arrêt burlesque de Boileau (qui paraît en août de cette année 1671) parle de "certains quidams factieux prenans les surnoms de Gassendistes et Cartésiens" (Malebranchistes et Pourchotistes seront ajoutés dans l'édition des CEuvres en 1701).

15 "Le Roi ayant appris que certains opinions que la Faculté de théologie avait censurée autrefois et que le Parlement avait défendu d'enseigner ni de publier, se répandent présentement non seulement dans l'Université, mais dans le reste de cette ville, et dans quelques autres du Royaume, soit par des étrangers, soit par des gens du dedans, voulant empêcher le cours de cette opinion qui pourrait porter quelque confusion dans l'explication de nos mystères, poussé de son zèle et de sa piété ordinaire, il m'a commandé de vous dire ses intentions. Le Roi vous demande, Messieurs, de faire en sorte que l'on n'enseigne point dans les Universités d'autre doctrine que celle qui est portée par les règlements et statuts de l'Université, et qu'on n'en mette rien dans les thèses, et laisse à votre prudence et à votre conduite de prendre les mesures nécessaires pour cela." (discours de Harlay au corps de Faculté, le 4 août 1671). 
Raimondo Capizucchi, ${ }^{16}$ les neotericorum quorumdam placita. ${ }^{17}$ Vers la fin de l'été, un mémoire anonyme, conservé à Rome aux Archives des carmes déchaux, demande de défendre la lecture des livres cartésiens. ${ }^{18}$ En septembre, le prémontré Libert De Pape, abbé de l'abbaye du Parc, ${ }^{19}$ fait la visitation de l'Université. Il note que le 27 septembre, un farouche adversaire du jansénisme naissant, Nicolas Du Bois, qui avait succédé en juin 1654 au philojanséniste Libert Froidmont dans la chaire d'Écriture sainte, ${ }^{20}$ se plaignait que le cartésianisme fût encore enseigné, nonobstant la condamnation romaine (dont il lui fait parvenir une copie le 2 octobre suivant). ${ }^{21}$ La nouvelle fut envoyée à Rome, au cardinal-neveu Paoluzzo Altieri, ${ }^{22}$ par l'internonce à Bruxelles, Ottavio Falconieri, plusieurs mois plus tard, le 24 octobre, avec quatorze extraits de thèses, que l'internonce estimait dignes de censure. Ces thèses de Universa philosophia avaient été soutenues le 17 juillet 1673 chez les oratoriens de Louvain.

L'affaire parait avoir fini sur une sorte de non-lieu. L'internonce demanda à la Faculté de théologie de ne recevoir Flémalle à la licence qu'après des explications sur ses thèses, ${ }^{23}$ ce qui fut fait le 28 mars $1674 .{ }^{24}$ Les enjeux s'étaient en effet déplacés entre temps, avec une décision de l'Inquisition romaine, communiquée le 2 octobre 1673. Il s'agissait là d'une procédure disciplinaire, et non pas d'une décision doctrinale. D'une part, elle ne pouvait s'appliquer que dans le domaine géographique où l'Inquisition romaine était reçue, c'est-à-dire à peu près les États pontificaux, et d'autre part, cette mesure réglementaire visait à refuser l'approbation d'imprimer aux auteurs niant la réalité des accidents et favorisant l'atomisme.

16 Capizucchi avait été nommé dans ces fonctions par Innocent X (1654), mais démis en 1663 par Alexandre VII. II venait d'être remis en charge par Clément X Altieri.

17 Documents publiés par Ceyssens, Lucien. La seconde période du jansénisme Rome-Bruxelles : Institute historique belge de Rome, 1968. t. I, p. 68 et sv.

18 Document publié par L. Ceyssens. Ibidem, p. 87.

19 Libert De Pape (1619-1682) était le frère de Léon-Jean De Pape (vers 1610-1685), chef et président du Conseil privé aux Pays-Bas (1671-1685); il fut chargé en 1674 de faire la visitatio de l'Université de Louvain pour vérifier si les ordonnances d'Albert et Isabelle y étaient touours en vigueur. Sur lui, notice (éd. Van Even) dans Biographie nationale, t. 5, Bruxelles, 1876, col. 615-618.

20 Sur Nicolas Du Bois (vers 1620-1696), notice (E.-H.-J. Reusens) in Biographie nationale, t. 5, Bruxelles, 1876, col. 196-204.

21 Ibidem, p. 84.

22 Le secrétaire d'État en titre était Francesco Nerli, mais le cardinale padrone Altieri exerçait les pouvoirs d'un premier ministre auprès de son oncle adoptif le pape Clément X, Emilio Altieri. II signe les courriers de la secrétairerie d'État et les lettres des nonces lui sont adressées.

23 Archives de l'Université de Louvain, Acta Facultatis S. Theologiae, vol. 389, f 147-148 (16 décembre 1673), document publié par Lucien Ceyssens. La seconde période du jansénisme, op. cit., p. 107-108.

24 Document cité ibidem, p. 170. 
Les enquêtes que nous venons de rappeler n'ont pas abouti à une nouvelle condamnation (il a suffi de confirmer celle de 1663), mais elles ont permis d'élargir les chefs d'accusation en motivant par les risques que la nouvelle physique faisait courir à l'explication de la transsubstantiation eucharistique ${ }^{25}$ une interdiction systématique du cartésianisme, condamné par l'Université d'Angers en 1675, puis par celle de Caen en 1677.

Le contexte est bien plus large que ce que Bayle veut bien indiquer: le souci de défendre l'orthodoxie catholique vient de Rome et du monarque français autant et plus que de Harlay. Enfin, les interdictions d'enseignements nouveaux étaient également partagées chez les jésuites comme chez les oratoriens, mais la structure plus souple de l'Oratoire, dont les maisons revendiquaient une certaine autonomie, n'avait pas permis une "mise au point" aussi rapide et décisive que dans la Compagnie de Jésus. Ce point est rappelé dans la circulaire de la XVI ${ }^{\mathrm{e}}$ Congrégation: "il sera libre aux théologiens de tenir et d'enseigneur soit aux nôtres soit aux non-théologiens [...] toutes les opinions qui sont reçues et librement enseignées dans l'Église, sans qu'on puisse leur imposer aucune nécessité de s'attacher aux unes plutôt qu'aux autres".

Les textes de l'Assemblée (lettre au roi, ordre de l'Assemblée pour être envoyé dans toutes les maisons de l'Oratoire et un extrait des actes de l'Assemblée) se trouvent aux Archives nationales (M. 218, XV, fos 39 sv; reproduit par André Robinet in Malebranche OC XVIII, p. 136-140). Le titre donné par Bayle (Concordat entre les Jésuites et les Pères de l'Oratoire) ne figure évidemment pas dans les originaux et copies de ces textes.

Enfin, ce que Bayle n'indique pas, les mêmes interdictions ont été portées, très tôt, dans les Universités calvinistes aux Pays-Bas, mettant à mal la libertas philosophandi. Le contexte est donc beaucoup plus général que ce que Bayle indique dans ses remarques.

b) Le deuxième texte: on se trouve ici devant un empilement de la critique "en abîme": au "concordat" sont jointes des réflexions inédites dont Bayle n'indique pas la paternité: "l'auteur dit franchement sa pensée" (dit Bayle, qui est lui-même en train de dissimuler), "et paraît fort habile homme".

Il y insiste sur deux points: d'une part, sur le caractère de "mauvais génie" de l'archevêque de Paris, à qui le roi laisserait toute latitude pour juger en matière doctrinale, et d'autre part sur l'omnipotence des jésuites, les oratoriens 
étant plus lâches que méchants. Le petit livre de Bayle a été important en histoire de la philosophie parce que beaucoup d'historiens ultérieurs ont reproduit ses éclaircissements, qui sont autant de traits polémiques.

c) Paru en 1680 sous le pseudonyme de La Ville, l'ouvrage du P. Louis Le Valois (1639-1700) attaquait vivement les Sentiments de M. Descartes touchant l'essence et la propriété des corps...: en clair, il reprochait à la physique cartésienne de ne pas pouvoir rendre compte de la transformation substantielle intervenant dans la doctrine catholique de l'Eucharistie, appelée transsubstantiation. Le titre complet de l'ouvrage décrit les trois parties: les "sentiments" de Descartes et des cartésiens (à la réserve du P. Maignan et de Cordemoy), la doctrine de l'Église, les erreurs de Calvin. L'ouvrage avait obtenu deux approbations (3 février et 24 mars 1678) et un privilège (concédé à un anonyme) le 7 novembre 1679; il avait été achevé d'imprimer le 8 janvier 1680 et était précédé d'une Épître "aux archevêques et évêques de France". Le Journal des savans en rendit compte dans son numéro IV du 12 février 1680, p. 41-45. Pierre Bayle en parle favorablement à Vincent Minutoli le 24 mars 1680 (Correspondance, t. III, p. 222): “c'est un traité qui est fort bien écrit, et l'auteur [...] prouve invinciblement ce qu'il veut prouver, car dans le fond, ce n'était guère difficile à faire".

Pour sa part, le jugement de Malebranche (lettre à P. Berrand, début 1680) est fort précieux: "ce livre quoique mal raisonné pourra faire bien du mal, car il est assez bien écrit, et il a des raisonnements spécieux" (OC XVIII, p. 169).

Bayle exagère l'effet de cet ouvrage, dans une mise en scène de terreur: "ce livre alarma toute la secte de ce philosophe", et entraînant une autocensure de Pierre-Sylvain Régis (retardant la publication de son Système de philosophie). Enfin, il brandit le spectre de l'hérésie: "chacun craignait de se voir obligé à la signature d'un formulaire ou d'être excommunié comme hérétique".

Autour de ce livre, qui était assez largement répandu (cinq exemplaires sont actuellement conservés dans les Bibliothèques publiques de France), Bayle donne trois pièces rares contribuant à sa discussion:

c1) l'Eclaircissement sur le livre de M. de La Ville, qui fut imprimé par son auteur, François Bernier, pour distinguer sa position, gassendiste, de celle des cartésiens. Bernier fit circuler sa brochure de manière discrète ("sourdement"). 
Il s'agit d'un texte, dont je ne connais qu'un exemplaire, un in- $4^{\circ}$ imprimé conservé dans un recueil du Cabinet des manuscrits de la BnF. La position de Bernier est fidéiste, et Bayle a dû être ravi d'y voir que l'impossibilité d'une explication physique de la transsubstantiation conduisait l'auteur à reconnaître que "quand même que par impossible la chose (= la transsubstantation) ne serait pas, ce ne serait au plus à notre égard qu'une simple erreur qui serait pardonnable, et qui ne saurait jamais nous être imputée".

c2) la Réponse de $M .{ }^{* * *}$ à une lettre de ses amis ...: cette pièce est très rare, le seul exemplaire connu de sa première édition, un opuscule in-12 de 24 pages, anonyme, s.l.n.d. (vers mi-1680) se trouve à la Bibliothèque de PortRoyal. Une copie manuscrite (peut-être antérieure à l'impression) se trouve à la $\mathrm{BnF}$ (fds fr 24500, f $\left.{ }^{\circ} 176-181\right) .{ }^{26}$ Le Mémoire pour expliquer la possibilité de la Transsubstantiation occupe les pages 18 à 24 de l'opuscule imprimé (ou les fos 181-183 de la copie manuscrite); il a connu seul un brillant destin, dans des copies manuscrites, puis à la suite du Traité de l'infini créé (Amsterdam, 1769). ${ }^{27}$ Un examen attentif des variantes entre les trois textes montre que Bayle n'a pas suivi l'imprimé: les deux corrections qu'il apporte au Mémoire (tête au lieu de terre, et nombres au lieu de membres) peuvent soit avoir été apportées par lui, soit provenir d'une copie (elles figurent dans la copie de la $\mathrm{BnF}$ ). Contrairement à la copie imprimée, Bayle n'attribue pas les deux textes à Malebranche (voir la discussion dans A. Robinet, $O C$ de Malebranche, t. XVII-1, p. 450 et sv).

c3) deux écrits latins de Bayle, une dissertation contre Ludovicus a Villa (1680) et XII thèses philosophiques de la même année, suivies de X corollaires. Bayle ajoute une note pour préciser que ces thèses ne sont pas là pour gonfler le volume, mais parce qu'elles ne sont pas sans rapport avec la dissertation et les réponses au P. Le Valois. La Dissertation et les thèses avaient été imprimées à Sedan durant l'été 1680. Leur étude détaillée reste à faire.

d) le dernier ouvrage du recueil est les Méditations de métaphysique de l'abbé François de Lanion, parues anonymes (s.1., 1678, in-24, 128 p.), ${ }^{28}$ puis

26 Édition critique par André Robinet, CEuvres complètes de Malebranche, t. XVII-1, p. 447.

27 Attribué à l'abbé Jean Terrasson, le Traité de l'infini créé a été republié, avec des documents, par Antonella Del Prete, en 2007, chez Honoré Champion à Paris.

28 L'exemplaire BnF R-43461 «manque en place". 
sous le pseudonyme de Guillaume Wander. ${ }^{29}$ Bayle doit en connaître l'auteur: “c'est apparemment un nom supposé, et c'est quelque Français, disciple de l'auteur de la Recherche de la Vérité, qui s'est ainsi travesti en Allemand ou en Flamand pour exciter moins de soupçons et de tempêtes". L'abbé de La Roque en parle dans le Journal des savans (XVII, 1678). L'ouvrage a fait l'objet de remarques de Leibniz (qui les attribuait à Malebranche, avec qui il échangea une correspondance à ce sujet). ${ }^{30}$ Une recension anonyme (due à Abraham Gaultier) parut dans la deuxième (et dernière) livraison du Mercure savant (Amsterdam, 1684), et Bayle réagit dans la première livraison des Nouvelles (mars 1684, p. 40-41). ${ }^{31}$ Lanion se liera avec Bayle dans les année 1690 lors d'un voyage aux Pays-Bas. ${ }^{32}$ La récente édition annotée de Jean-Christophe Bardout restitue à ce texte toute sa profondeur malebranchiste.

Notre propos de conclusion porte sur l'Avis au lecteur. L'intérêt de ce recueil réside peut-être moins dans les pièces qui le composent que précisément dans son organisation. Il nous semble que ce recueil possède plusieurs traits typiques de l'écriture de Bayle: le souci de la librairie et de la liberté d'impression, lié à des intérêts commerciaux: ce qui est interdit se vend mieux que ce qui est permis. Il insiste sur le secret, "la dérobée". Les oratoriens, Bernier, Malebranche, Lanion: leurs textes donnent chair à une charpente organisée par Bayle, avec son avis au lecteur, ses commentaires du "concordat" et ses textes latins.

Surtout, Bayle soigne le paratexte: faux-titre donné aux Actes des oratoriens, page de titre entière pour le traité de Lanion et, enfin, son "avis au lecteur", dont le titre lui-même est déjà habile: l'avis au lecteur introduit une distance entre le recueil de texte et sa présentation qui est différente de toute introduction, préface, avant-propos ou liminaire. L'Ad lectorem, c'est la prise de parole de l'imprimeur, du libraire ou de l'éditeur. L'éditeur anonyme se distancie avec d'autant plus de vigueur qu'une bonne partie des textes anonymes du recueil sont de sa plume.

29 Outre l'édition par Adolphe Garnier dans les CEuvres de Descartes (citée n. 1), le texte a aussi été publié par Félix-Sébastien Feuillet de Conches (attribué à Malebranche) en 1841. II a enfin été réédité, avec une excellente présentation et annotation, par J.-Chr. Bardout (Paris: Vrin, 2009). J.-Chr. Bardout a retrouvé à la Landesbibliothek de Hanovre un exemplaire contenant une onzième Méditation, dont Bayle n'a pas eu connaissance.

30 Sämtliche Schriften und Briefe, VI, 4, Deutsche Akademie der Wissenschaften (éd.), p. 1778-1783 et Malebranche, CEuvres complètes, éd. A. Robinet, t. XVIII, p. 161-162 (voir aussi Robinet, A. Malebranche et Leibniz. Relations personnelles. Paris: Vrin, 1955).

31 Voir la note de J.-Chr. Bardout, op. cit., p. 8, avec les indications bibliographiques aux ouvrages d'Olivier Bloch.

32 Mentionné par Labrousse, E. Pierre Bayle. Paris : Albin Michel, $2^{e}$ éd. 1966. p. 185. 
Sous l'apparent irénisme (“on n'a nullement en vue d'aigrir les catholiques romains contre les cartésiens de leur communion", une affirmation réitérée dans les $N R L$ ), Bayle met en place une attaque de fond contre la croyance catholique. Sous couleur de dénoncer la perséuction dont les cartésiens peuvent être victimes, il établit l'impossibilité de la croyance catholique. L'alternative est de rejeter la nouvelle philosophie ou, si on l'adopte, de rejeter le concile de Trente et le dogme catholique.

\section{Références bibliographiques}

Académie royale des sciences, des lettres et des beaux-arts de Belgique. Biographie nationale, t. 5. Bruxelles, 1876, col. 615-618.

ARMOGATHE, J.-R. - CARRAUD, Vincent. "La première condamnation des Euvres de Descartes, d'après des documents inédits aux Archives du Saint-Office". Nouvelles de la République des lettres (Naples), 2001-II, p.103-137.

BAYLE, Pierre (éd.). Recueil de quelques pièces curieuses concernant la philosophie de M. Descartes. Amsterdam: Henri Desbordes, 1684.

BERNIER, François. Eclaircissement sur le livre de M. de La Ville. [s.l.n.d.].

BOILEAU-DESPRÉAUX, Nicolas. Euvres. Paris: D. Thierry, 1701.

BOILEAU-DESPRÉAUX, Nicolas. Euvres. Éd. par Saint-Marc. Amsterdam: P. J. Changuion, 1772.

BOST, Hubert. Pierre Bayle historien, critique et moraliste. Turnhout: Brepols, 2006.

CEYSSENS, Lucien. La seconde période du jansénisme. Bruxelles-Rome : Institute historique belge de Rome, 1968, t. I.

CLAVE, Étienne de. Nouvelle lumière philosophique. Édité par Bernard Joly. Paris: Fayard (coll. Corpus), 2000.

CORAS, Jacques de. David, ou la vertu couronnée. Paris: C. Angot, 1665.

CORAS, Jacques de. Jonas ou Ninive pénitente. Paris: C. Angot, 1663.

DECKER, Léger-Charles. Cartesius seipsum destruens. Louvain : P. Sasseni, 1675.

DESCARTES, René. CEuvres philosophiques, éd. Adrien Garnier, t. IV. Paris: Hachette, 1835.

FÉRET, P. La Faculté de théologie de Paris et ses docteurs les plus célèbres. Époque moderne, t. VII: XVIII siècle: Revue littéraire. Paris: A. Picard et fils, 1910.

KAHN, Didier. "La condamnation des thèses d'Antoine de Villon et Étienne de Clave contre Aristote, Paracelse et les 'cabalistes' (1624)". Revue d'histoire des sciences (2002), 55, 2, p. 143-198.

LA MARE, Nicolas de. Traité de la police. T. I, Paris: J. et P. Cot, 1705.

LABROUSSE, Elisabeth. Pierre Bayle, $2^{\mathrm{e}}$ éd. Paris: Albin Michel, 1996.

LANION, François de. Méditations de métaphysique. [s.1.] 1678. 
LANION, François de. Méditations sur la métaphysique. [...] textes édités par Jean-Christophe Bardout et Kristell Trego; présentés et annotés par Jean-Christophe Bardout. Paris: Vrin, 2009.

LE VALOIS, Louis. Sentimens de M. Descartes touchant l'essence et les propriétés du corps, opposés à la doctrine de l'Église, et conformes aux erreurs de Calvin sur le sujet de l'Eucharistie. Avec une dissertation sur la prétendue possibilité des choses impossibles..., Paris: E. Michallet, 1680.

LEIBNIZ, Gottfried Wilhelm. Sämtliche Schriften und Briefe, VI, 4, Deutsche Akademie der Wissenschaften éd., Berlin: Akademie Verlag, 1999.

MALEBRANCHE, Nicolas. Euvres complètes. Édition critique par André Robinet, t. XVII-1, Paris: Vrin, 1960; t. XVIII, Paris: Vrin, 1978.

[Malebranche, Nicolas de.] Méditations métaphysiques et Correspondance [...] avec J.-J. Dortous de Mairan sur des sujets de métaphysique, publiées pour la première fois sur les manuscrits originaux (par F. Feuillet de Conches). Paris: H. Delloye, 1841.

MCKENNA, Antony et al. éd. Correspondance de Pierre Bayle, t. III. Oxford: Voltaire Foundation, 2004.

Réponse de M. *** à une lettre de ses amis [s.l.n.d., vers mi-1680].

ROBINET, André. Malebranche et Leibniz. Relations personnelles, Paris: Vrin, 1955.

SAUVY, Anne. Livres saisis à Paris entre 1678 et 1701, La Haye: M. Nijhoff, 1972. SORTAIS, Gaston. Le Cartésianisme chez les jésuites français au XVII et au XVIII siècle. Paris: Beauchesne, 1929.

TERRASSON, Jean. Traité de l'infini créé. Amsterdam, 1769. Rééd. par Antonella Del Prete, Paris: Honoré Champion 2007. 